\title{
Portal Annular Pancreas (PAP): an Underestimated Devil in Pancreatic Surgery-Systematic Review of Literature and Case Report
}

\author{
Saneya Pandrowala ${ }^{1} \cdot$ Aamir Parray $^{1} \cdot$ Vikram Chaudhari $^{1} \cdot$ Shailesh V. Shrikhande ${ }^{1} \cdot$ Manish S. Bhandare $^{1}$ (D)
}

Received: 6 November 2020 / Accepted: 15 January 2021 / Published online: 8 February 2021

(C) 2021 The Society for Surgery of the Alimentary Tract

\begin{abstract}
Introduction Portal annular pancreas (PAP) is an anatomic variation due to aberrant fusion of the ventral and dorsal pancreatic buds around the portal vein. In this article, we present a case report with a systematic review of literature of patients undergoing major pancreatic surgery with associated PAP. We also intend to discuss and suggest possible surgical strategies to minimise major postoperative complications.

Methods A systematic literature search was conducted using the terms "circumportal," "periportal," "pancreas," "annular pancreas," "portal annular pancreas" and "pancreas anomaly." All articles describing portal annular pancreas with surgical resection were included.

Results We identified a total of 53 patients of PAP from 29 articles, who underwent pancreatic resection with a median age of 65 years. POPF (postoperative pancreatic fistula) was demonstrated in $42.55 \%$ of patients and $34 \%$ had CR (clinically relevant)POPF. Following pancreaticoduodenectomy, pancreatic stump was reconstructed in all patients with either pancreaticojejunostomy or pancreaticogastrostomy. Standard line of pancreatic transection, i.e., division of anteportal portion at the pancreatic neck and stapling of the retroportal process, resulted in $71 \%$ incidence of CR-POPF, whereas it was only $16 \%$ when extended resection was performed to achieve single pancreatic stump and $12.5 \%$ when retroportal portion was sutured or ligated. Amongst distal pancreatic resections, $66 \%$ had POPF and 33\% developed CR-POPF.

Conclusion It is of utmost importance for pancreatic surgeons to diligently look for and identify PAP in the preoperative imaging. Additional imaging in the form of MRCP helps to define abnormal pancreatic ductal anatomy. Surgeons need to be cognisant of pancreatic stump management in patients with PAP to reduce associated higher rates of POPF.
\end{abstract}

Keywords Portal annular pancreas $\cdot$ PAP $\cdot$ Annular pancreas $\cdot$ Circumportal pancreas $\cdot$ POPF $\cdot$ Pancreatic stump

\section{Introduction}

Portal annular pancreas (PAP) is an anatomic variation resulting due to aberrant fusion of the ventral and dorsal pancreatic buds around the portal vein. PAP was first described by Suguira as the hypertrophic uncinate process. ${ }^{1}$ Often, it is incidentally detected on CT (computed tomography) scan done for other purposes and the reported incidence ranges from 0.8 to $2.5 \% .^{2-5}$ However, its true incidence remains unknown as PAP is generally an underreported anomaly as it can

Manish S. Bhandare

manishbhandare@gmail.com

1 Gastrointestinal and Hepato-Pancreato-Biliary Service, Department of Surgical Oncology, Tata Memorial Hospital,

Mumbai, Maharashtra 400012, India be easily missed or mistaken for locally advanced focal lesion of the head or uncinate process of the pancreas. On retrospectively analysing radiology reports of 1000 patients, PAP was reported in one of $25(4 \%)$ scans. ${ }^{5}$ PAP has been classified by Joseph et al. ${ }^{6}$ in 3 types: type 1 is the fusion of the ventral bud of the pancreas with the body and retroportal MPD (main pancreatic duct), type 2 is type 1 associated with pancreas divisum and type 3 is the portal vein encasement by the uncinate process with a normal anteportal MPD. Karasaki et al. ${ }^{4}$ have subdivided each type (A, B and C) depending on the relation to the portal confluence as suprasplenic, infrasplenic and mixed type (Fig. 1).

It is crucial to preoperatively identify this anomaly to assess the ductal anatomy better, preferably by MRI with MRCP (magnetic resonance imaging with magnetic resonance cholangiopancreatography) together with contrast CT scan, so as to plan the operative procedure appropriately. The 
Fig. 1. PAP classification by Karasaki et al. ${ }^{4}$ : a normal uncinate process, $\mathbf{b}$ suprasplenic fusion of pancreas around portal vein as shown by arrowhead, c infrasplenic fusion and $\mathbf{d}$ mixed fusion

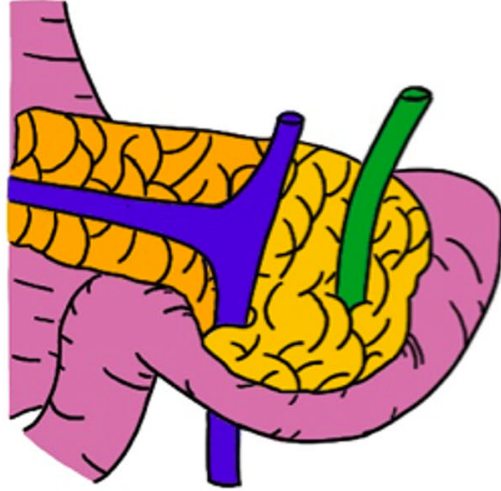

a

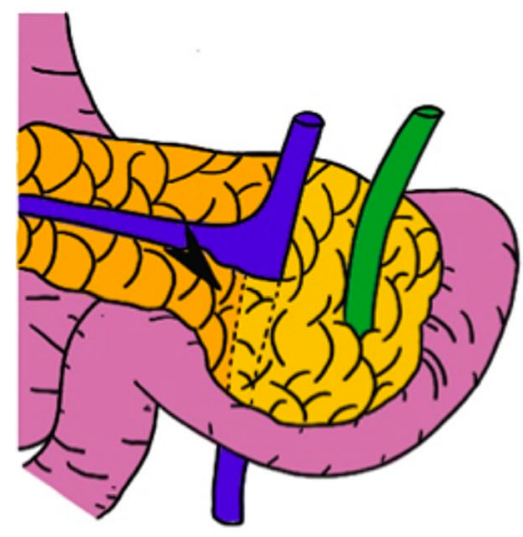

C

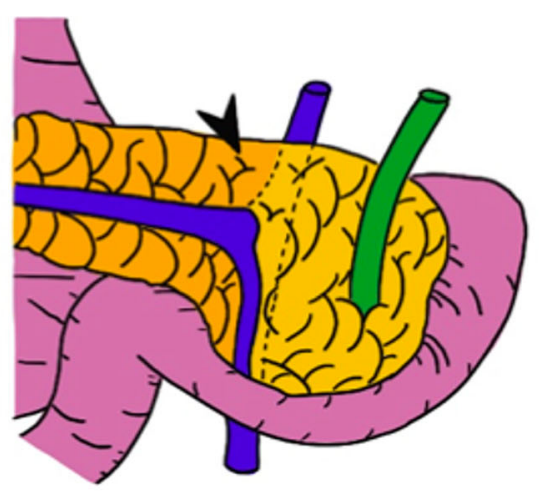

b

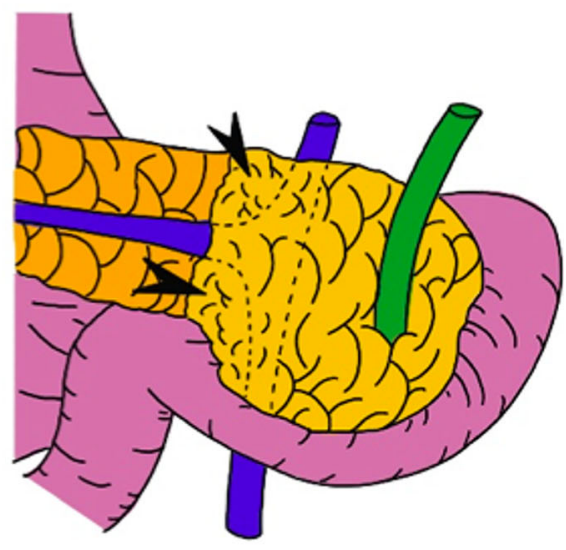

d complexity associated with surgery in PAP is due to the varying MPD anatomy and two pancreatic resection surfaces and thus is associated with a high incidence of postoperative pancreatic fistula (POPF).

In this article, we present a case report with systematic review of literature, highlighting the significance of PAP in pancreatic surgery and suggesting possible surgical strategies to decrease major postoperative complications.

\section{Materials and Methods}

A systematic literature search was conducted by using the data available from PubMed central (https:/www.ncbi.nlm.nih. gov/pmc/) from May, 1987 to June, 2020 by two authors independently as per PRISMA (Preferred Reporting Items for Systematic Reviews and Meta-Analyses) guidelines. All articles without language restrictions were searched using the terms "circumportal," "periportal," "pancreas," "annular pancreas," "portal annular pancreas" and "pancreas anomaly." All articles describing portal annular pancreas with surgical resection involving the pancreas or pancreatic surgery were included. After excluding articles from titles and abstracts, eligible articles were assessed with complete manuscripts for details regarding the type of PAP, primary tumour, surgery performed, techniques for management of pancreatic stump and POPF. References of retrieved manuscripts were also assessed for other eligible articles.

Along with the cases reported in literature, a patient with PAP who recently underwent pancreatic surgery at Tata Memorial Hospital, Mumbai, India, was also included in the analysis and is presented as a case report. Signed informed consent was obtained from this patient for any surgical and clinical procedure. The study protocol was in accordance with the ethical standards of the institutional research committee and the 1964 Helsinki Declaration with its later amendments. Since this was a retrospective observational study with review of literature, formal consent for this study was not required and no approval of the institutional research committee was needed.

\section{Case Report}

A 58-year-old gentleman without any comorbidity presented to our institute with history of biliary stenting and cholangitis. He had undergone ERCP (endoscopic retrograde cholangiopancreatography) and plastic stent placement 2 years 
Fig. 2. Preoperative CT scan of a 58-year-old male with periampullary cancer showing type 3A PAP. a Anteportal pancreas and retroportal pancreas. $\mathbf{b}$ MPD in anteportal pancreas (arrow)
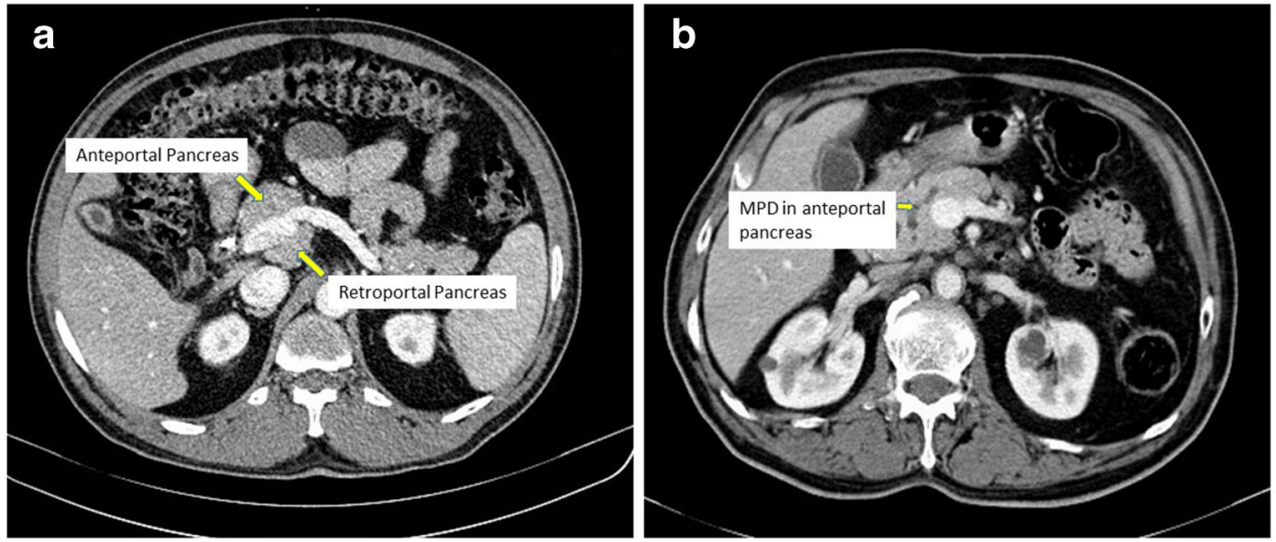

back. Brush cytology and biopsy from the ampullary region was negative for malignancy at that time. Due to repeated attacks of cholangitis, stent exchange was performed twice after the initial procedure. The subsequent periampullary biopsy was suggestive of adenocarcinoma, following which he was referred to our centre. CA 19.9 level was $13002 \mathrm{IU} / \mathrm{ml}$, and contrast-enhanced CT scan (triphasic) revealed dilatation of common bile duct with stent in situ and bulky uncinate process. Pancreatic duct was not dilated; no definite lesion was appreciated in periampullary region. Patient was planned for pancreaticoduodenectomy (PD) after preoperative COVID testing (surgery was performed during the coronavirus pandemic) as per institutional protocol. Intraoperative findings were suggestive of type 3/A PAP with no duct identified in the annular portion which was corroborated by retrospectively visualising the CT images as depicted in Fig. 2. The annular portion was $2.5-\mathrm{cm}$ thick and was completely excised. It was closed with interrupted PDS 4-0 sutures and was not included in the anastomosis, while the pancreas stump (anterior to portal vein) was reconstructed by pancreaticojejunostomy. This intraoperative surprise lead to increased blood loss $(2000 \mathrm{ml})$, unanticipated difficult surgery, prolonged duration of surgery (360 $\mathrm{min}$ ) and uncertainty regarding the possibility of a major duct in the retroportal pancreas. Postoperatively the patient developed pancreatic fistula on POD (postoperative day) 2 with left drain contents showing clear pancreatic fluid which was initially managed conservatively. Patient was clinically doing well and a precautionary CT scan done on POD 5 suggested a posterior pancreatic leak (Fig. 3CT image showing air speck behind PJ) without any undrained collection. On POD 6, he developed fever and small abdominal wound gape with purulent bilious discharge. His general condition remained stable. Percutaneous transhepatic biliary drainage (PTBD) with interno-external catheter was performed to gain early control over the biliary and pancreatic fistula. The cholangiogram showed a point leak from hepatico-jejunostomy from postero-medial surface. The leak resolved gradually with drainage and appropriate antibiotics as per culture sensitivity. PTBD was clamped on POD 18 and removed on POD 20. He was discharged on POD 23 tolerating oral feeds with left side drain in situ which was removed on POD 31. He was planned for adjuvant chemotherapy and is currently on gemcitabine-based chemotherapy in view of histopathology suggestive of ampulla of Vater adenocarcinoma with R0 resection and 5 out of 17 nodes positive for metastasis (pT3N2).

\section{Results of Literature Review}

We identified 2484 records by the database and 164 records through other mentioned sources (Fig. 4). After excluding 158
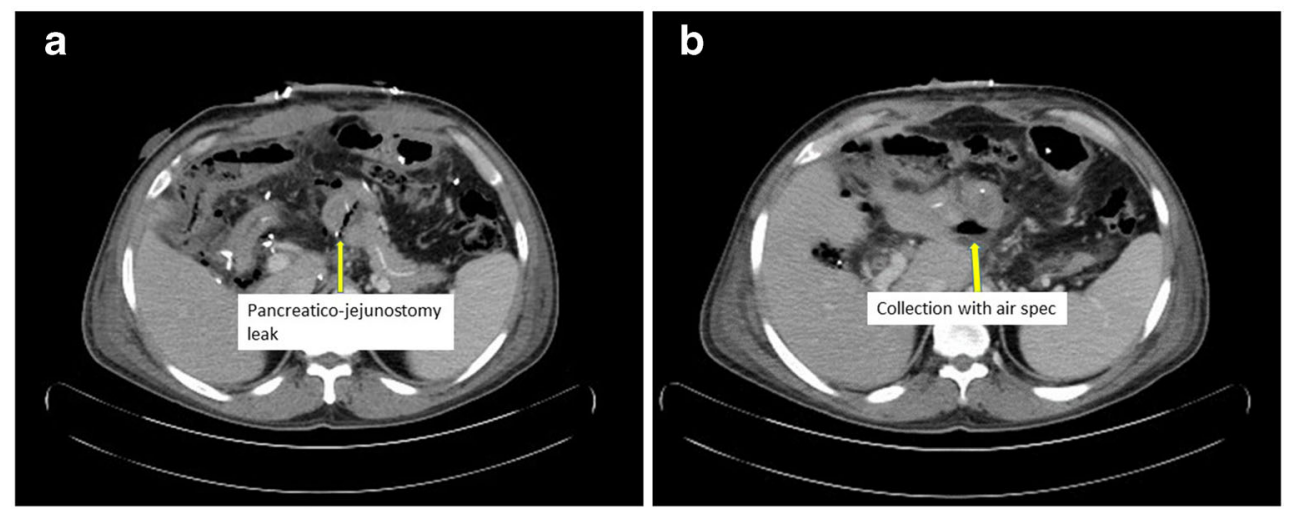

Fig. 3. Postoperative day 5 of pancreaticoduodenectomy CT showing collection with air spec behind (arrow) pancreaticojejunostomy suggestive of pancreatico-jejunostomy leak 
Fig. 4. Consort chart

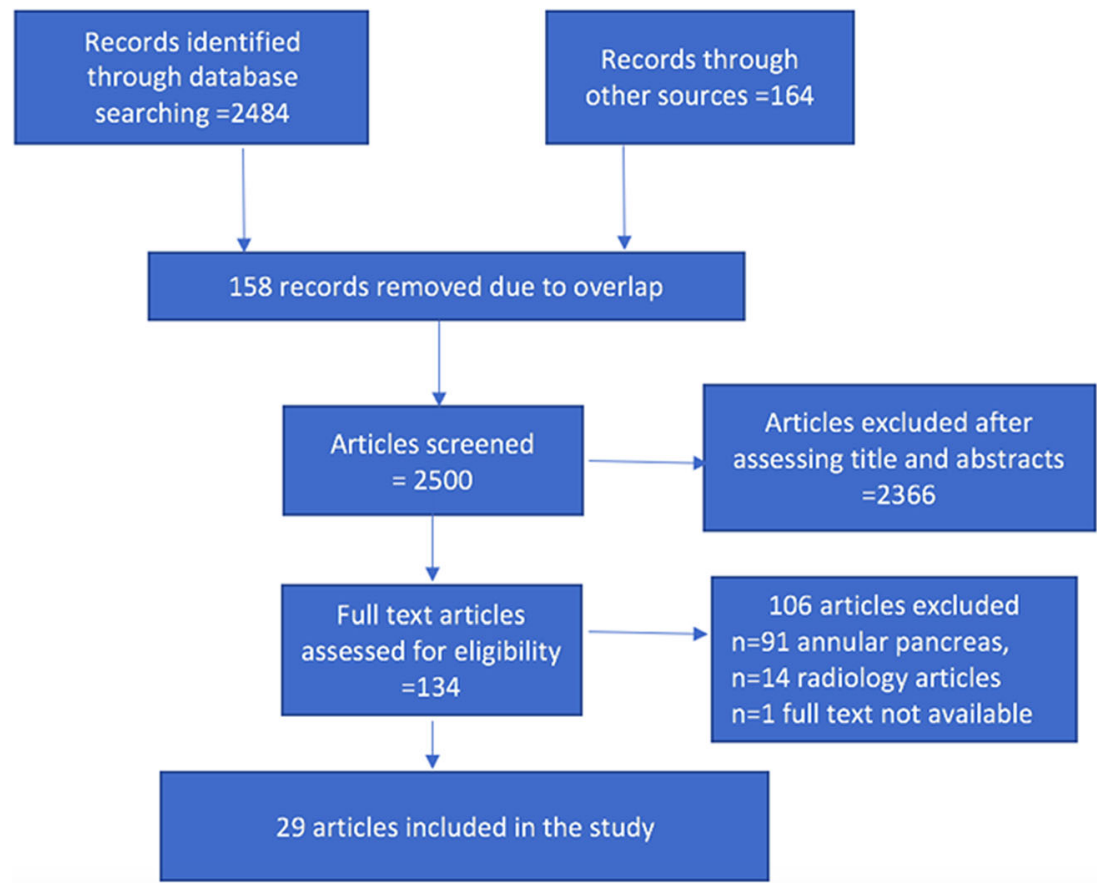

duplicate records, 2500 articles were screened by title and abstracts following which 134 full-text articles were assessed for eligibility. We included 29 articles gathering data of 52 patients with PAP who underwent pancreatic surgery. Including our case, a total of 53 patients of PAP with pancreatic resection or surgery aged 39-84 years (median 65 years) were analysed. There was a slight preponderance for males $(56 \%$ - 29 out of 52) with $44 \%$ being women. Primary histopathology varied as seen in Table 1, with majority of cases presenting with pancreatic ductal adenocarcinoma $(n=11)$, ampulla of Vater tumours $(n=10)$ and bile duct lesions $(n=9)$. Operative procedures included 40 pancreaticoduodenectomies (39: open, 1: laparoscopic), 3 total pancreatectomies, 2 radical antegrade modular pancreaticosplenectomies (RAMPS; 1: open, 1: laparoscopic), 2 multivisceral resections, 1 central pancreatectomy, 1 distal pancreaticosplenectomy (DPS), 1 laparoscopic distal pancreatectomy spleen preserving (DPSP), one hepatopancreaticoduodenectomy and one distal pancreatectomy with coeliac axis resection (DP-CAR).

As per the classification of PAP by Joseph and Karasaki, there were 25 cases (49\%) with type 3/A PAP (Table 1) which was the most common type, unlike the observation from a study with retrospective evaluation of CT scans, where type 3/C has been identified more commonly. ${ }^{3}$ Only 3 cases were identified with retroportal MPD (type 1) and 5 cases with retroportal MPD combined with pancreatic divisum (type 2). Thirty patients $(64 \%)$ had suprasplenic fusion of pancreatic parenchyma (type A), 10 (21\%) had infrasplenic (type B) and $7(15 \%)$ were mixed fusion (type C).

Amongst the 53 cases, data regarding POPF was available for all but three patients and since 3 patients underwent total pancreatectomy, POPF rate was calculated from 47 cases. POPF was demonstrated in $42.55 \%$ of patients (20 out of 47 ) and $34 \%$ (16 out of 47) had clinically relevant leak (Grade B-15, Grade C-1). Following pancreaticoduodenectomy, pancreatic stump was reconstructed in all patients with either pancreaticojejunostomy or pancreaticogastrostomy. As can be noted in Table 2, patients with standard line of pancreatic transection at the neck of the anteportal portion and stapling of the retroportal process resulted in $71 \%$ incidence of CR-POPF, whereas it was only $16 \%$, when an extended resection was performed to achieve a single pancreatic stump and $12.5 \%$ when retroportal portion was sutured or ligated. Amongst 6 distal pancreatic resections, 66\% had POPF (4 out 6) and $33 \%$ developed CR-POPF (2 out of 6 ). Since these numbers are small, it cannot help in definitive decision making as regards the effective technique for pancreatic stump management; however, this highlights implications of PAP in pancreatic surgery with associated high rate of POPF.

\section{Discussion}

Despite being a well-known and well-described entity, PAP continues to be under-reported and under-recognised. Pancreatic surgery in patients with PAP is associated with higher rates of POPF. Systematic review by Harnoss et al. included 21 studies with POPF rate in patients with PAP (12 pancreaticoduodenectomies and 3 distal pancreatectomies) being $46.7 \%$ as per ISGPS classification. ${ }^{3}$ Analogously, based on the data available, we had 29 studies with 53 patients and a $42.55 \%$ rate of POPF and $34 \%$ CR-POPF. The study by 
Table 1 Published cases with pancreatic resection in PAP

\begin{tabular}{|c|c|c|c|c|c|c|c|c|}
\hline $\begin{array}{l}\text { Sr. } \\
\text { no. }\end{array}$ & Author & Patient & Primary tumour & Surgery & $\begin{array}{l}\text { PAP } \\
\text { typel }\end{array}$ & Non MPD pancreas stump ${ }^{*}$ & $\begin{array}{l}\mathrm{PJ}: \\
\mathrm{PG}^{\mathrm{bc}}\end{array}$ & POPF \\
\hline 1 & Suguira et al. ${ }^{1}$ & $51 / \mathrm{F}$ & $\begin{array}{l}\text { Inflammatory head } \\
\text { mass }\end{array}$ & $\mathrm{PD}^{\mathrm{a}}$ & 3/B & Interrupted sutures & $\mathrm{PJ}^{\mathrm{c}}$ & No \\
\hline 2 & Hiroshi et al. ${ }^{7}$ & $76 / \mathrm{M}$ & Metastatic $\mathrm{RCC}^{\mathrm{p}}$ & $\begin{array}{l}\text { Central } \\
\text { pancreatectomy }\end{array}$ & $3 / \mathrm{A}$ & $\begin{array}{l}\text { Proximal: stapled } \\
\text { Distal: } \mathrm{PJ}^{\mathrm{c}}\end{array}$ & $\mathrm{PJ}^{\mathrm{c}}$ & $\mathrm{B}$ \\
\hline 3 & Kawamoto et al. $^{8}$ & $76 / \mathrm{F}$ & PDAC $^{\mathrm{n}}$ & $\mathrm{PD}^{\mathrm{a}}$ & $1 / \mathrm{A}$ & Mobilised- $>\mathrm{PJ}^{\mathrm{c}}$ & $\mathrm{PJ}^{\mathrm{c}}$ & No \\
\hline 4 & Tousif et al. ${ }^{9}$ & $\begin{array}{l}81 / \mathrm{F} \\
76 / \mathrm{M}\end{array}$ & $\begin{array}{l}\text { IPMN }^{\mathrm{m}} \\
\text { Bile duct }\end{array}$ & $\begin{array}{l}\mathrm{TP}^{\mathrm{d}} \\
\mathrm{PD}^{\mathrm{a}}\end{array}$ & $\begin{array}{l}3 / \mathrm{C} \\
3 / \mathrm{C}\end{array}$ & $\begin{array}{l}\text { Uncinate stapled } \\
\text { Sutured }\end{array}$ & - & NA \\
\hline 5 & Hamanaka et al. ${ }^{10}$ & $59 / \mathrm{M}$ & Ampullary & $\mathrm{PD}^{\mathrm{a}}$ & $3 / \mathrm{C}$ & $\mathrm{NA}$ & - & NA \\
\hline 6 & Hashimoto et al. ${ }^{11}$ & $39 / \mathrm{F}$ & Mucinous neoplasm & DPS & 2 & Interrupted sutures & - & A \\
\hline 7 & Balila et al. $^{12}$ & $72 / \mathrm{M}$ & Duodenal GIST & $\mathrm{PD}^{\mathrm{a}}$ & $3 / \mathrm{A}$ & NA & $\mathrm{PJ}^{\mathrm{c}}$ & A \\
\hline 8 & Ishigami et al. ${ }^{2}$ & $\begin{array}{l}45 / \mathrm{F} \\
80 / \mathrm{M} \\
65 / \mathrm{M}\end{array}$ & $\begin{array}{l}\text { Insulinoma } \\
\text { IPMN }^{\mathrm{m}} \\
\text { PDAC }^{\mathrm{n}}\end{array}$ & $\begin{array}{l}\mathrm{PD}^{\mathrm{a}} \\
\mathrm{PD}^{\mathrm{a}}->\text { cyst excision } \\
\mathrm{PD}^{\mathrm{a}}\end{array}$ & $\begin{array}{l}\text { NA } \\
\text { NA } \\
3 / A\end{array}$ & $\mathrm{NA}$ & - & No \\
\hline 9 & Jang et al. ${ }^{13}$ & $\begin{array}{l}71 / \mathrm{M} \\
74 / \mathrm{M}\end{array}$ & $\begin{array}{l}\mathrm{IPMN}^{\mathrm{m}} \\
\mathrm{IPMN}^{\mathrm{m}}\end{array}$ & $\begin{array}{l}\text { PD }^{\mathrm{a}} \\
\text { Lap RAMPS }\end{array}$ & $\begin{array}{l}3 / \mathrm{A} \\
\mathrm{C}\end{array}$ & $\begin{array}{l}\text { Stapled } \\
\text { NA }\end{array}$ & $\begin{array}{l}\mathrm{PJ}^{\mathrm{c}} \\
-\end{array}$ & $\begin{array}{l}\mathrm{B} \\
\mathrm{A}\end{array}$ \\
\hline 10 & Joseph et al. ${ }^{6}$ & $51 / \mathrm{M}$ & Ampullary & $\mathrm{PD}^{\mathrm{a}}$ & $2 / \mathrm{A}$ & $\begin{array}{l}\text { Interrupted sutures, side to } \\
\text { side } \mathrm{PJ}^{\mathrm{c}}\end{array}$ & $\mathrm{PJ}^{\mathrm{c}}$ & No \\
\hline 11 & Kobayashi et al. ${ }^{14}$ & $61 / \mathrm{F}$ & Ampullary & $\mathrm{PD}^{\mathrm{a}}$ & $3 / \mathrm{A}$ & Interrupted sutures & $\mathrm{PJ}^{\mathrm{c}}$ & No \\
\hline 12 & Kuriyama et al. ${ }^{15}$ & $47 / \mathrm{F}$ & $\begin{array}{c}\text { Serous cystic } \\
\text { neoplasm }\end{array}$ & Lap DPSP & $3 / \mathrm{A}$ & Stapled & - & $\mathrm{B}$ \\
\hline 13 & Izuishi et al. ${ }^{16}$ & $50 / \mathrm{M}$ & Bile duct & $\mathrm{PD}^{\mathrm{a}}$ & $3 / \mathrm{C}$ & Extended resection & $\mathrm{PJ}^{\mathrm{c}}$ & No \\
\hline 14 & Marjanovic et al. ${ }^{17}$ & $65 / \mathrm{M}$ & Ca stomach & Multivisceral $^{\mathrm{e}}$ & $3 / \mathrm{A}$ & Stapled & $\mathrm{PJ}^{\mathrm{c}}$ & $\mathrm{B}$ \\
\hline 15 & Baskaran et al. ${ }^{18}$ & 47/M & Ampullary & $\mathrm{PD}^{\mathrm{a}}$ & 3 & Sutures & $\mathrm{PJ}^{\mathrm{c}}$ & No \\
\hline 16 & Karasaki et al. ${ }^{4}$ & $73 / \mathrm{F}$ & Bile duct & $\mathrm{PD}^{\mathrm{a}}$ & $3 / \mathrm{C}$ & NA & $\mathrm{PG}^{\mathrm{b}}$ & $\mathrm{B}$ \\
\hline 17 & Zimmitti et al. $^{19}$ & 71 & Ampullary & Lap PD ${ }^{a}$ & $3 / \mathrm{B}$ & Extended resection & $\mathrm{PG}^{\mathrm{b}}$ & No \\
\hline 18 & Muto et al. ${ }^{20}$ & $45 / \mathrm{F}$ & Insulinoma & $\mathrm{PD}^{\mathrm{a}}$ & 2 & Extended resection & $\mathrm{PJ}^{\mathrm{c}}$ & No \\
\hline 19 & Kiuchi et al. ${ }^{21}$ & $\begin{array}{l}78 / \mathrm{M} \\
76 / \mathrm{M} \\
55 / \mathrm{M} \\
74 / \mathrm{M} \\
66 / \mathrm{M} \\
65 / \mathrm{M} \\
79 / \mathrm{F}\end{array}$ & $\begin{array}{l}\text { PDAC }^{\mathrm{n}} \\
\text { Bile duct } \\
\text { Ampullary } \\
\text { Bile duct } \\
\text { Duodenal } \\
\text { IPMN }^{\mathrm{m}} \\
\text { Ampullary }\end{array}$ & $\begin{array}{l}\mathrm{PD}^{\mathrm{a}} \\
\mathrm{PD}^{\mathrm{a}} \mathrm{PD}^{\mathrm{a}} \mathrm{PD}^{\mathrm{a}} \mathrm{PD}^{\mathrm{a}} \mathrm{PD}^{\mathrm{a}} \\
\mathrm{PD}^{\mathrm{a}}\end{array}$ & $\begin{array}{l}3 / \mathrm{B} \\
3 / \mathrm{B} \\
3 / \mathrm{B} \\
3 / \mathrm{B} \\
3 / \mathrm{B} \\
3 / \mathrm{B} \\
3 / \mathrm{B}\end{array}$ & $\begin{array}{l}\text { Cautery } \\
\text { Stapled } \\
\text { Cautery } \\
\text { Stapled } \\
\text { Stapled } \\
\text { Stapled } \\
\text { Stapled }\end{array}$ & $\begin{array}{l}\mathrm{PJ}^{\mathrm{c}} \\
\mathrm{PJ}^{\mathrm{c}} \\
\mathrm{PJ}^{\mathrm{c}} \\
\mathrm{PJ}^{\mathrm{c}} \\
\mathrm{PJ}^{\mathrm{c}} \\
\mathrm{PJ}^{\mathrm{c}} \\
\mathrm{PJ}^{\mathrm{c}}\end{array}$ & $\begin{array}{l}\text { No } \\
\text { BCBBBNo }\end{array}$ \\
\hline 20 & Matsumoto et al. ${ }^{22}$ & $81 / \mathrm{F}$ & Ampullary & $\mathrm{PD}^{\mathrm{a}}$ & 1 & Extended resection & & No \\
\hline 21 & Matsumoto et al. ${ }^{23}$ & $78 / \mathrm{M}$ & Duodenal & $\mathrm{PD}^{\mathrm{a}}$ & $3 / \mathrm{A}$ & Both stumps-PG ${ }^{\mathrm{b}}$ & $P G^{b}$ & No \\
\hline 22 & Pardiwala et al. ${ }^{24}$ & $81 / \mathrm{F}$ & Duodenal & $\mathrm{PD}^{\mathrm{a}}$ & $3 / \mathrm{A}$ & Ligated & $\mathrm{PJ}^{\mathrm{c}}$ & No \\
\hline 23 & Yuan et al. ${ }^{25}$ & $74 / \mathrm{M}$ & $\mathrm{PDAC}^{\mathrm{n}}$ & RAMPS $^{\mathrm{f}}$ & $3 / \mathrm{B}$ & NA & - & No \\
\hline 24 & Shonaka et al. ${ }^{26}$ & $53 / \mathrm{M}$ & PDAC $^{\mathrm{n}}$ & $\mathrm{PD}^{\mathrm{a}}$ & $3 / \mathrm{A}$ & Both stumps-PG ${ }^{\mathrm{b}}$ & $\mathrm{PG}^{\mathrm{b}}$ & $\mathrm{A}$ \\
\hline 25 & Zhang et al. ${ }^{27}$ & $66 / \mathrm{M}$ & $\mathrm{IPMN}^{\mathrm{m}}$ & $\mathrm{PD}^{\mathrm{a}}$ & $3 / \mathrm{C}$ & Continuous sutures & $\mathrm{PJ}^{\mathrm{c}}$ & No \\
\hline 26 & Ohtsuka et al. ${ }^{28}$ & $\begin{array}{l}66 / \mathrm{M} \\
64 / \mathrm{M} \\
65 / \mathrm{F} \\
63 / \mathrm{M} \\
61 / \mathrm{F} \\
76 / \mathrm{F} \\
46 / \mathrm{M} \\
84 / \mathrm{F} \\
77 / \mathrm{F}\end{array}$ & $\begin{array}{l}\text { PDAC }^{\mathrm{n}} \\
\text { IPMN }^{\mathrm{m}} \\
\text { Bile duct } \\
\text { NET }^{\mathrm{o}} \\
\text { PDAC }^{\mathrm{n}} \\
\text { Bile duct } \\
\text { Bile duct } \\
\text { Bile duct }^{\text {PDAC }^{\mathrm{n}}}\end{array}$ & $\begin{array}{l}\mathrm{PD}^{\mathrm{a}} \\
\mathrm{PD}^{\mathrm{a}} \\
\mathrm{HPD}^{\mathrm{h}} \\
\mathrm{DP}^{\mathrm{i}} \\
\mathrm{DP}^{\mathrm{h}} \mathrm{CAR}^{\mathrm{ik}} \\
\mathrm{PD}^{\mathrm{a}} \\
\mathrm{PD}^{\mathrm{a}} \\
\mathrm{PD}^{\mathrm{a}} \\
\mathrm{PD}^{\mathrm{a}}\end{array}$ & $\begin{array}{l}3 / \mathrm{A} \\
3 / \mathrm{A} \\
3 / \mathrm{A} \\
3 / \mathrm{A} \\
3 / \mathrm{A} \\
3 / \mathrm{A} \\
3 / \mathrm{A} \\
3 / \mathrm{A} \\
3 / \mathrm{A}\end{array}$ & $\begin{array}{l}\text { Stapled } \\
\text { Extended resection } \\
\text { Extended resection } \\
\text { Stapled } \\
\text { Stapled } \\
\text { Stapled } \\
\text { Stapled } \\
\text { Extended resection } \\
\text { Extended resection }\end{array}$ & - & $\begin{array}{l}\text { No } \\
\text { B } \\
\text { No } \\
\text { No } \\
\text { B } \\
\text { No } \\
\text { B } \\
\text { B } \\
\text { No }\end{array}$ \\
\hline 27 & Harnoss et al. ${ }^{3}$ & $48 / \mathrm{F}$ & Suprarenal & Multivisceral $^{\mathrm{e}}$ & $3 / \mathrm{A}$ & NA & & $\mathrm{B}$ \\
\hline 28 & Luu et al. ${ }^{29}$ & $\begin{array}{l}81 / \mathrm{M} \\
49 / \mathrm{F} \\
60 / \mathrm{M} \\
65 / \mathrm{F} \\
73 / \mathrm{F} \\
55 / \mathrm{F}\end{array}$ & $\begin{array}{l}\text { Ampullary } \\
\text { IPMN }^{\mathrm{m}} \\
\text { Chronic pancreatitis } \\
\text { Ampullary } \\
\text { PDAC }^{\mathrm{n}} \\
\text { Serous cystadenoma }^{\text {ser }}\end{array}$ & $\begin{array}{l}\mathrm{PD}^{\mathrm{a}} \\
\mathrm{PD}^{\mathrm{a}} \\
\mathrm{PD}^{\mathrm{a}} \\
\mathrm{TP}^{\mathrm{d}} \\
\mathrm{PD}^{\mathrm{a}} \\
\mathrm{TP}^{\mathrm{d}}\end{array}$ & $\begin{array}{l}2 / \mathrm{A} \\
3 / \mathrm{A} \\
2 / \mathrm{A} \\
3 / \mathrm{A} \\
3 / \mathrm{A} \\
3 / \mathrm{A}\end{array}$ & $\begin{array}{l}\text { Extended resection } \\
\text { Extended resection } \\
\text { Extended resection } \\
\text { NA } \\
\text { Extended resection } \\
\text { NA }\end{array}$ & $\begin{array}{l}\mathrm{PJ}^{\mathrm{c}} \\
\mathrm{PJ}^{\mathrm{c}} \\
\mathrm{PJ}^{\mathrm{c}} \\
- \\
\mathrm{PJ}^{\mathrm{c}} \\
-\end{array}$ & No \\
\hline
\end{tabular}


Table 1 (continued)

\begin{tabular}{|c|c|c|c|c|c|c|c|c|}
\hline $\begin{array}{l}\text { Sr. } \\
\text { no. }\end{array}$ & Author & Patient & Primary tumour & Surgery & $\begin{array}{l}\text { PAP } \\
\text { typel }\end{array}$ & Non MPD pancreas stump ${ }^{*}$ & $\begin{array}{l}\mathrm{PJ}: \mathrm{P}- \\
\mathrm{G}^{\mathrm{bc}}\end{array}$ & POPF \\
\hline 29 & Narita et al. ${ }^{30}$ & $72 / \mathrm{F}$ & PDAC $^{\text {n }}$ & $\mathrm{PD}^{\mathrm{a}}$ & $1 / \mathrm{A}$ & Both stumps-PG ${ }^{\mathrm{b}}$ & $\mathrm{PG}^{\mathrm{b}}$ & NA \\
\hline 30 & $\begin{array}{l}\text { Case from present } \\
\text { study }\end{array}$ & $58 / \mathrm{M}$ & $\mathrm{PDAC}^{\mathrm{n}}$ & $\mathrm{PD}^{\mathrm{a}}$ & $3 / \mathrm{A}$ & Interrupted sutures & $\mathrm{PJ}^{\mathrm{c}}$ & $\mathrm{B}$ \\
\hline
\end{tabular}

*Non MPD pancreas stump - pancreas stump in PAP without the main pancreatic duct and its management

${ }^{\text {a }} P D$ pancreaticoduodenectomy

${ }^{\mathrm{b}} P G$ pancreaticogastrostomy

${ }^{\mathrm{c}} P J$ pancreaticojejunostomy

${ }^{\mathrm{d}} T P$ total pancreatectomy

${ }^{\mathrm{e}}$ Multivisceral 14-subtotal gastrectomy+ right hemicolectomy+PD, 27-DP+splenectomy+L nephrouretrectomy+ hemicolectomy

${ }^{\mathrm{f}} R A M P S$ radical antergrade modular pancreaticosplenectomy

${ }^{\mathrm{h}} H P D$ hepato-pancreaticoduodnectomy

${ }^{\mathrm{i}} D P$ distal pancreatecomy

${ }^{\mathrm{j}} D P S P$ distal pancreatectomy spleen preserving

${ }^{\mathrm{k}} C A R$ coeliac axis resection

${ }^{1} P A P$ type Joseph/Karasaki

${ }^{\mathrm{n}} P D A C$ pancreatic ductal adenocarcinoma

${ }^{\mathrm{m}} I P M N$ intraductal papillary mucinous neoplasm

${ }^{\circ}$ NET neuroendocrine tumour

${ }^{\mathrm{p}} R C C$ renal cell carcinoma.

Harnoss et al. included 17 cases who underwent pancreatic resection and preoperative diagnosis was missed in almost half of the patients on imaging. Even in our patient, the anomaly was not reported on presurgery evaluation scan (triphasic CT scan).

When PAP is suspected/identified on CT scan, MRI with MRCP becomes an essential tool to delineate pancreatic ductal anatomy. Preoperative or intraoperative identification of PAP is extremely essential so as to adapt to a different strategy during pancreatic resection as well as stump reconstruction. These patients are clearly at a higher risk of developing POPF due to the varied anatomy, and hence, alteration in the surgical techniques can help in managing the pancreatic stump better with favourable postoperative outcomes. Following surgical strategies can be adapted in patients with PAP.

\section{During Pancreaticoduodenectomy (PD)}

The technical aspects of surgery relate to the pancreatic remnant posterior to the portal vein or SMV. The non-dominant cutting plane, i.e., the plane without MPD can be either sutured or stapled. ${ }^{23,28}$ It has also been suggested that PG with invagination of the two resected pancreatic planes together into the stomach after PD helps to minimise resected volume
Table 2 Pancreatic transection type association with POPF after pancreaticoduodenectomy

\begin{tabular}{lll}
\hline Pancreatic transection after PD with PAP $(n=42)$ & Number of patients & CR-POPF rate \\
\hline Extended resection & 12 & $16 \%(2 / 12)$ \\
Both stumps-PG/PJ & 4 & $0 \%(0 / 3,1-\mathrm{NA})$ \\
Stapled retroportal portion & 15 & $71.4 \%(10 / 14,1-\mathrm{NA})$ \\
Sutured/ligated retroportal portion & $9(8 / 1)$ & $12.5 \%(1 / 8,1-\mathrm{NA})$ \\
Cautery for retroportal portion & 2 & $50 \%(1 / 2)$ \\
\hline
\end{tabular}

${ }^{\text {a }} P G$ pancreaticogastrostomy

${ }^{\mathrm{b}} P J$ pancreaticojejunostomy

${ }^{\mathrm{c}} C R-P O P F$ clinically relevant postoperative pancreatic fistula 
Table 3. Type of PAP and proposed surgical management

\begin{tabular}{lc}
\hline Type of PAP & Proposed surgical management \\
\hline $\begin{array}{c}\text { Type 1 and 2, i.e., retroportal MPD with } \\
\text { or without accessory anteportal MPD }\end{array}$ & $\begin{array}{c}\text { Extended resection-excising entire PAP } \\
\text { to achieve single pancreatic stump } \\
\text { Type 3, i.e., anteportal MPD alone } \\
\text { Standard plane of resection with suturing } \\
\text { of the retroportal portion }\end{array}$ \\
\hline
\end{tabular}

of the pancreas as well as possibly reduce chance of POPF. ${ }^{22}$ Interestingly, either a PG or PJ with invagination of both stumps have not resulted in CR-POPF as per the available data. If the MPD is in the retroportal pancreas or there are two ducts then it has been suggested that the transection plane needs to be extended so as to avoid two pancreatic cut surfaces and avoid two anastomoses which are associated with increased incidence of POPF. ${ }^{6}$ However, this technique needs more dissection and resection of larger pancreatic volume that might cause deterioration of the remnant pancreatic function. The present study demonstrates a lower leak rate with extended resection or suturing of the pancreatic stump as opposed to stapling. This is possibly due to direct visualisation of any major duct which can be closed or anastomosed. However, in our case, we sutured the non-dominant cutting plane and still our patient experienced POPF ISGPS grade B. Although extended pancreatic resection to achieve single cut surface did not result in POPF, these patients had longer hospital stays between 18 and 32 days. ${ }^{8,19,20}$

\section{During Distal Pancreatic Resections}

In distal pancreatectomy, the thickness of the pancreas at the staple line along with the technique of gradual compression during stapling affects the risk of POPF ${ }^{31,32}$ Since in PAP two margins are present which increases the thickness, it is advisable to staple the ventral and dorsal margins separately than to perform a single staple line at the region of SMA. ${ }^{15,31}$ This has been demonstrated by Kuriyama et al. in their report of laparoscopic spleen preserving distal pancreatectomy where they have transected the pancreas with two separate staple lines for the ventral and dorsal portions owing to thickness of more than $12 \mathrm{~mm} .{ }^{15}$ Although the patient developed grade B POPF, she was discharged on POD 9.

As per the results of this review, extended resection or suturing/ligation of the retroportal portion, both remain viable options while dealing with pancreatic stump after PD in PAP. Extended resections can be challenging since the pancreatic tissue is likely to creep along the posterior surface of hepatic artery and celiac axis in suprasplenic fusion type PAP and along SMA in infrasplenic type. Extended resection can result in higher operative blood loss and higher risk of secondary bleeding complications (PPH) in the event of a POPF. Hence, preoperative identification of ductal anatomy should be of utmost importance so as to classify patients with PAP by
Josephs' classification accurately. In case, the diagnosis of PAP is missed preoperatively; intraoperative ultrasound should be used to define MPD anatomy. We propose an individualised management for pancreatic stump after PD according to the Josephs' classification (Table 3).

Thus, tailoring the type of resection can prevent extended surgery in type 3 PAP with associated higher bleeding risk and future pancreatic insufficiency, yet offering a safe postoperative course with acceptable fistula rates.

For DPS/DPSP/RAMPS, in view of thicker pancreas at the region of SMA, all types should be managed by two separate stapler lines for the ventral and dorsal portions.

We recognise the limitations of this study since this is a retrospective review of literature with small numbers of patients; however, every pancreatic surgeon must be familiar with identification and management of PAP.

\section{Conclusion}

It is of utmost importance for pancreatic surgeons to diligently look for and identify PAP in the preoperative imaging, in order to understand the ductal anatomy and communication. If this opportunity to recognise PAP is missed, it can lead to inappropriate management of the pancreatic stump resulting in higher rates of POPF and associated devastating consequences. Surgical strategies to minimise the rate of CRPF during PD should involve extended resections or suture closure of the retroportal tissue, depending on the PAP type.

\section{Declarations}

Conflict of Interest The authors declare no competing interests.

\section{References}

1. Sugiura Y, Shima S, Yonekawa H, Yoshizumi Y, Ohtsuka H, Ogata T. The hypertrophic uncinate process of the pancreas wrapping the superior mesenteric vein and artery-A case report. The Japanese journal of surgery. 1987;17(3):182-5.

2. Ishigami K, Tajima T, Nishie A, Asayama Y, Kakihara D, Nakayama T, Shirabe K, Taketomi A, Nakamura M, Takahata S, Ito $\mathrm{T}$. The prevalence of circumportal pancreas as shown by multidetector-row computed tomography. Insights into imaging. 2011;2(4):409-14. 
3. Harnoss JM, Harnoss JC, Diener MK, Contin P, Ulrich AB, Büchler MW, Schmitz-Winnenthal FH. Portal annular pancreas: a systematic review of a clinical challenge. Pancreas. 2014;43(7): 981.

4. Karasaki H, Mizukami Y, Ishizaki A, Goto J, Yoshikawa D, Kino S, Tokusashi Y, Miyokawa N, Yamada T, Kono T, Kasai S. Portal annular pancreas, a notable pancreatic malformation: frequency, morphology, and implications for pancreatic surgery. Surgery. 2009;146(3):515-8.

5. John R, Simon B, Eapen A, Putta T, Joseph P, Raju RS, Rymbai ML, Vyas F. Portal Annular Pancreas: An Underrecognized Variant, Prevalence, and Clinical Relevance. Tropical Gastroenterology. 2020;40(4):132-6.

6. Joseph P, Raju RS, Vyas FL, Eapen A, Sitaram V. Portal annular pancreas. A rare variant and a new classification. JOP. Journal of the Pancreas. 2010;11(5):453-5.

7. Yamaguchi H, Kimura Y, Nagayama M, Imamura M, Tanaka S, Yoshida M, Yoshida E, Fujino H, Machiki T, Miyanishi K, Mizuguchi T. Central pancreatectomy in portal annular pancreas for metastatic renal cell carcinoma: a case report. World journal of surgical oncology. 2019;17(1):1-6.

8. Kawamoto H, Fujikawa T, Tanaka A. Successful resection of pancreatic head cancer in a patient with circumportal pancreas: a case report with technical consideration. Innovative surgical sciences. 2017;2(1):33-7.

9. Kabir T, Xuan ZT, Chung AY. Circumportal pancreas: A report of two cases. Annals of Hepato-Biliary-Pancreatic Surgery. 2019;23(3):300-4.

10. Hamanaka Y, Evans J, Sagar G, Neoptolemos JP. Complete pancreatic encasement of the proximal hepatic portal vein: a previously undescribed congenital anomaly. British journal of surgery. 1997;84(6):785.

11. Hashimoto Y, Ross AS, Traverso LW. Circumportal pancreas with retroportal main pancreatic duct. Pancreas. 2009;38(6):713-5.

12. Balila RM, Saad EA, Babiker MA, Mahadi SE. Duodenal GIST with Associated Portal Annular Pancreas: A Report of Rare Pancreatic Anomaly.Sudan Medical Journal. 2017;11(5563):1-4.

13. Jang JY, Chung YE, Kang CM, Choi SH, Hwang HK, Lee WJ. Two cases of portal annular pancreas. The Korean Journal of Gastroenterology. 2012;60(1):52-5.

14. Kobayashi S, Honda G, Kurata M, Okuda Y, Tsuruta K. Pancreaticoduodenectomy in portal annular pancreas: report of a case. Surgery today. 2013;43(8):926-9.

15. Kuriyama N, Hatanaka T, Gyoten K, Hayasaki A, Fujii T, Iizawa Y, Kato H, Murata Y, Tanemura A, Kishiwada M, Sakurai H. How to divide the pancreatic parenchyma in patients with a portal annular pancreas: laparoscopic spleen-preserving distal pancreatectomy for serous cystic neoplasms. Surgical Case Reports. 2020;6:1-5.

16. Izuishi K, Wakabayashi H, Usuki H, Suzuki Y. Anomalous annular pancreas surrounding the superior mesenteric vessel. ANZ journal of surgery. 2010;80(5):376-7.

17. Marjanovic G, Obermaier R, Benz S, Bley T, Juettner E, Hopt UT, Adam U. Complete pancreatic encasement of the portal veinsurgical implications of an extremely rare anomaly. Langenbeck's archives of surgery. 2007;392(4):489-91.

18. Baskaran D, Gomathi S, Balamourougan K, Ramkumar G, Jigish R, Aniruthan D, Sistla SC. Portal Annular Pancreas: Case Report of a Rare Anomaly. Cureus. 2018; 10(3): e2366
19. Zimmitti G, Manzoni A, Ramera M, Villanacci A, Sega V, Treppiedi E, Guerini F, Garatti M, Codignola C, Rosso E. Management of portal annular pancreas during laparoscopic pancreaticoduodenectomy. Journal of minimal access surgery. 2018;14(4):354.

20. Muto J, Mano Y, Harada N, Uchiyama H, Yoshizumi T, Taketomi A, Shirabe K, Maehara Y. Additional resection of the pancreas body prevents postoperative pancreas fistula in patients with portal annular pancreas who undergo pancreaticoduodenectomy. Case Reports in Gastroenterology. 2012;6(1):131-4.

21. Kiuchi R, Mizuno T, Okamura Y, Sugiura T, Kanemoto H, Uesaka $\mathrm{K}$. Circumportal pancreas-a hazardous anomaly in pancreatic surgery. HPB. 2018;20(5):385-91

22. Matsumoto I, Shinzeki M, Fukumoto T, Ku Y. An extremely rare portal annular pancreas for pancreaticoduodenectomy with a special note on the pancreatic duct management in the dorsal pancreas. Surgery 2013;153:434-436.

23. Matsumoto I, Kamei K, Satoi S, Nakai T, Takeyama Y. Pancreaticogastrostomy Prevents Postoperative Pancreatic Fistula of Portal Annular Pancreas During Pancreaticoduodenectomy. International Surgery. 2016;101(11-12):550-3.

24. Pardiwala KH, Thorlacius H. Operative management of portal annular pancreas during pancreaticoduodenectomy. Surgery 2016;159:972-974.

25. Yuan H, Wu P, Chen J, Lu Z, Chen L, Wei J, Guo F, Cai B, Yin J, $\mathrm{Xu}$ D, Jiang K. Radical antegrade modular pancreatosplenectomy for adenocarcinomaof the body of the pancreas in a patient with portal annular pancreas, aberrant hepatic artery, and absence of the celiac trunk: A case report. Medicine. 2017;96(48):e8738.

26. Shonaka $\mathrm{T}$, Inagaki $\mathrm{M}$, Akabane $\mathrm{H}$, Yanagida $\mathrm{N}$, Shomura $\mathrm{H}$, Kudo T, Orimo T, Oikawa F, Aiyama T, Yanagawa N, Oikawa K. Pancreatoduodenectomy for circumportal pancreas accompanying the retroportal pancreatic duct: a case report and review of the literature. Clinical journal of gastroenterology. 2012;5(5):332-5.

27. Zhang B, Yang XJ, Qian ZY. Pancreatic Fusion Abnormality Found During a Whipple Procedure. Gastroenterology. 2017;153(6):e16-7.

28. Ohtsuka T, Mori Y, Ishigami K, Fujimoto T, Miyasaka Y, Nakata K, Ohuchida K, Nagai E, Oda Y, Shimizu S, Nakamura M. Clinical significance of circumportal pancreas, a rare congenital anomaly, in pancreatectomy. The American Journal of Surgery. 2017;214(2): 267-72.

29. Luu AM, Braumann C, Herzog T, Janot M, Uhl W, Chromik AM. Circumportal Pancreas - a Must Know Pancreatic Anomaly for the Pancreatic Surgeon. Journal of Gastrointestinal Surgery. 2017;21(2):344-51.

30. Narita M, Hata H, Ikai I. Portal annular pancreas: An unusual pancreatic anomaly. Journal of visceral surgery. 2016;153(2):153.

31. Mendoza AS 3rd, Han HS, Ahn S, Yoon YS, Cho JY, Choi Y. Predictive factors associated with postoperative pancreatic fistula after laparoscopic distal pancreatectomy: a 10-year single-institution experience. Surg Endosc. 2016; 30:649-56.

32. Kondo N, Uemura K, Nakagawa N, Okada K, Kuroda S, Sudo T, Hadano N, Matstukawa H, Satoh D, Sasaki M, Abe T. A multicenter, randomized, controlled trial comparing reinforced staplers with bare staplers during distal pancreatectomy (HiSCO-07 Trial). Annals of surgical oncology. 2019;26(5):1519-27.

Publisher's Note Springer Nature remains neutral with regard to jurisdictional claims in published maps and institutional affiliations. 